\section{Jahrestagung der DGP}

Wir möchten Sie an dieser Stelle auf die Phlebo-Arena - ein neues Format im Rahmen der diesjährigen Jahrestagung aufmerksam machen.

Im Rahmen der Phlebo-Arena bietet sich während des gesamten Kongresses die Möglichkeit, sich zu treffen und zu diskutieren. Verschiedene Gruppen innerhalb unserer Gesellschaft stellen sich vor, die Arbeitsgemeinschaften nutzen den Raum für ihren informellen und wissenschaftlichen Austausch. Höhepunkt des ArenenProgramms wird ein täglicher „Battle“ sein, in dem sich kurz und knapp in der Mittagszeit zwei Kontrahenten mit einem "Ringrichter" einen wissenschaftlichen Schlagabtausch liefern - lassen Sie sich überraschen von dieser etwas anderen Art des wissenschaftlichen Programms.
60. Jahrestagung der Deutschen

Gesellschaft für Phlebologie

26. - 29.09.2018
Stadthalle Bielefeld, Willy Brandt Platz 1, 33602 Bielefeld
Tagungspräsident

PD Dr. med. Helger Stege, Detmold

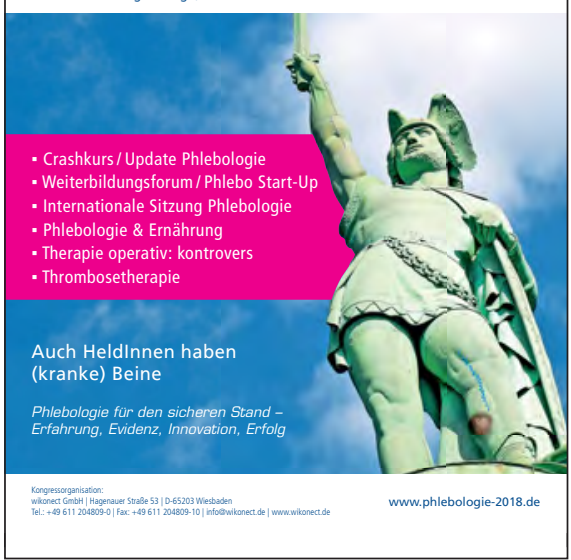

Im Rahmen der 60. Jahrestagung der DGP vom 28.9.2018, 16.45 Uhr in Bielefeld

\title{
Einladung Mitgliederversammlung
}

\section{Sehr geehrte Frau Kollegin, sehr geehrter Herr Kollege, \\ im Namen unseres Präsidenten, Herrn Professor Dr. med. M. Stücker, lade ich Sie herzlich zur Mitgliederversammlung ein.}

Ort: Stadthalle Bielefeld, Datum: Freitag, 28. September 2018, 16.45 - 18.15 Uhr, Kleiner Saal
Tagesordnung:

- Gedenken der Verstorbenen

1. Genehmigung der Tagesordnung

2. Genehmigung des Protokolls der Mitgliederversammlung vom 22.9.2017 in Stuttgart (veröffentlicht in Phlebologie 6/2017)

3. Bericht des Präsidenten

4. Bericht der Generalsekretärin

5. Bericht des Schatzmeisters

6. a) Bericht der Kassenprüfer

b) Entlastung des Vorstandes
7. Wahlen

8. Berichte der Kongresspräsidenten

$-2019$

$-2020$

9. Zusatzbezeichnung Phlebologie

10. Forschungsförderungskommission und Forschungsbericht

11. Bericht aus dem Berufsverband

12. Leitlinien

13. Fortbildungsakademie der Deutschen Gesellschaft für Phlebologie

14. Bericht aus der Zeitschrift Phlebologie

15. Berichte aus den Kommissionen und AG's

16. Verschiedenes

Dr. med. E. Mendoza, Generalsekretärin
Treffen der AG Hämodynamik in Bielefeld am 28.09.2018, 10.30 Uhr. Der Raum wird durch Aushang bekannt gegeben. Auch die AG Endo trifft sich am Freitag, vormittags um 9.00 Uhr. Auch hier beachten Sie bitte die gesonderte Ausschilderung. Die AG Thrombose, die
AG Sklerotherapie und die AG Arbeitsmedizin treffen sich ebenfalls in Bielefeld. Ihre Treffen finden jeweils in der Phlebo-Arena statt. Die genauen Zeiten erfahren Sie vor Ort in einem gesonderten Flyer.
Verantwortlich für Mitteilungen der DGP: Dr. med. Erika Mendoza 